\title{
Assessment of inlet efficiency through a 3D simulation: numerical and experimental comparison
}

\author{
Manuel Gómez, Joan Recasens, Beniamino Russo \\ and Eduardo Martínez-Gomariz
}

\begin{abstract}
Inlet efficiency is a requirement for characterizing the flow transfers between surface and sewer flow during rain events. The dual drainage approach is based on the joint analysis of both upper and lower drainage levels, and the flow transfer is one of the relevant elements to define properly this joint behaviour. This paper presents the results of an experimental and numerical investigation about the inlet efficiency definition. A full scale (1:1) test platform located in the Technical University of Catalonia (UPC) reproduces both the runoff process in streets and the water entering the inlet. Data from tests performed on this platform allow the inlet efficiency to be estimated as a function of significant hydraulic and geometrical parameters. A reproduction of these tests through a numerical three-dimensional code (Flow3D) has been carried out simulating this type of flow by solving the RANS equations. The aim of the work was to reproduce the hydraulic performance of a previously tested grated inlet under several flow and geometric conditions using Flow-3D as a virtual laboratory. This will allow inlet efficiencies to be obtained without previous experimental tests. Moreover, the 3D model allows a better understanding of the hydraulics of the flow interception and the flow patterns

Q2 approaching the inlet.
\end{abstract}

Key words |

\section{INTRODUCTION}

During extreme rainfall events uncontrolled amounts of runoff that are not captured by the surface drainage inlet system. This can produce serious hazard conditions for pedestrians and vehicles. These can cause significant direct and indirect damages related, respectively, to the assets exposed to flooding and the activities that can be affected (e.g. traffic disruption). In this context, it is clear that the surface drainage system needs to be composed by a series of welllocated inlets. In order to determine correctly the inlet spacing need to guarantee safe conditions for pedestrian and vehicles during a storm event and to avoid economic losses, a full knowledge of the hydraulic behaviour of the flow approaching the inlet and its performance is essential.

So, the drainage system must then be understood not only as a number of pipes but as a set of streets, grates and pipes which interact. This is the concept of dual drainage, which considers the stormwater flowing in two parallel layers, one formed by underground sewers and the

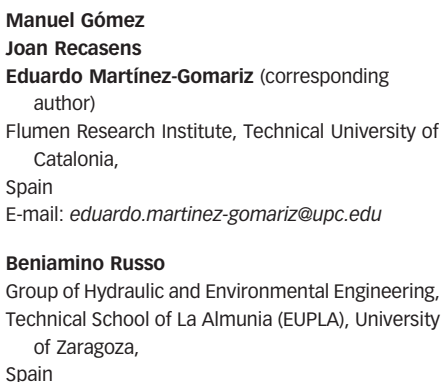


However, to do this, it is necessary to evaluate and quantify the water exchange through the grate. This flow collection for a rain event depends on several factors such as the grate size, its geometry, the street geometry and spatial density of the inlets. Following this idea, a better understanding of the hydraulics of this element and the interactions between surface flow and the underground drainage systems is clearly needed. Some institutions or companies have promoted the experimental testing of inlets. Examples of this type of study can be found in the Q4 technical literature like Li (1956); Argue (1986); Spaliviero

Q5 \& May (1998) or Gómez \& Russo (20II), but manufacturers or municipal technicians do not have the resources to test all the different grates that they use. Therefore, one option could be the use of a 3D code to simulate the flow behaviour of the grates, in some ways like a virtual laboratory.

In recent years, several experiments using 3D simu-

Q6 lations have been carried out (Djordjević et al. 2013; Lopes et al. 2013), but these have focused on the hydraulics of the inlet manhole with poor regard to the efficiency of the grated inlet defined as the ratio between the intercepted flow and the flow approaching the inlet.

Lopes et al. (20I3) developed a 3D approach using OpenFOAM $^{\circledR}$ code to study the surcharge flow in a gully, especially focusing on the jet characterization and the height it reaches above the gully. The code and a very detailed mesh allowed the researchers to obtain good similarity between numerical and experimental results. Djordjević et al. (20I3) carried out a 3D study of the flow pattern near one grate with the same code, considering the flow in both directions, entering the grate and leaving the sewer.

Noted above, these experiments did not focus on the hydraulic efficiency of the grates, so, in this context, a 3D computer code to reproduce grated inlet performance was applied and the results are shown in this paper. This paper focuses on the 3D modelling of one specific inlet, the socalled 'Barcelona1', one of the most common grates in the metropolitan area of Barcelona. The inlet was previously tested in the UPC hydraulic laboratory which enables us to compare the numerical results with the experimental values.

The main purpose of this paper is to reproduce the results of an experimental campaign carried out some years ago about the characterization of the hydraulic efficiency of some inlets commonly used in Barcelona (Spain) using a three-dimensional computational model. The results of this experimental campaign were published in 2011 (Gómez \& Russo 20II). In this paper, the authors proposed an empirical relationship to obtain the hydraulic efficiency as a function of inlet and street flow characteristics (flow approaching the inlet $Q$ and upstream flow depth $y$ ). Hydraulic efficiency $\left(E^{\prime}\right)$ was defined as the ratio between the captured flow by the inlet $Q_{\text {int }}$ and the approaching flow $Q$.

$E^{\prime}=A\left(\frac{Q}{y}\right)^{-B}$

where $E^{\prime}$ is the hydraulic efficiency of the inlet for a $3 \mathrm{~m}$ wide lane (defined as a decimal between 0 and 1 ), $Q$ is the discharge approaching the inlet circulating through the model $\left(\mathrm{m}^{3} / \mathrm{s}\right)$, $\mathrm{y}$ is the flow depth measured at the curb immediately upstream the inlet (m), $A$ and $\mathrm{B}$ are two empirical coefficients specific for each inlet grate.

\section{PHYSICAL MODEL: EXPERIMENTAL SET UP}

The experimental set up consists in a platform of $5.5 \mathrm{~m}$ length and $4 \mathrm{~m}$ width where a full scale inlet can be installed. As noted above, in this case the study focuses on the grate 'Barcelona1'. Figure 1 shows the main characteristics of this grate, located next to the curb.

The experimental platform (Figure 2) is supported at three points and by adjusting their heights the longitudinal and transverse slopes required can be established. The range of these values is from 0 to $4 \%$ for the transverse slope $\left(S_{T}\right)$, and between 0 and $10 \%$ for the longitudinal slope $\left(S_{L}\right)$. Upstream, a water tank provides a smooth flow that produces a one-dimensional flow boundary condition. The flow rate tested varies from 25 to $200 \mathrm{l} / \mathrm{s}$ in steady flow conditions. The input flow $(Q)$ is measured through an electromagnetic flow meter (with $11 / \mathrm{s}$ accuracy) while the intercepted flow ( $\left.\mathrm{Q}_{\text {int }}\right)$ (captured by the grate) is measured by a V-notch weir. These values are defined by the laboratory pumping capacity, but are of similar order of magnitude of those found in the streets of Barcelona (design storm for 10 years return period considers $56 \mathrm{~mm}$ in one hour, with peak rainfall intensities during 5 minutes of $180 \mathrm{~mm} / \mathrm{h}$ ).

For different combinations of circulating flow $Q(25 \mathrm{l} / \mathrm{s}$, $50 \mathrm{l} / \mathrm{s}, 100 \mathrm{l} / \mathrm{s}, 150 \mathrm{l} / \mathrm{s}$ and $200 \mathrm{l} / \mathrm{s})$, and longitudinal $(0 \%$, $0.5 \%, 1 \%, 2 \%, 4 \%, 6 \%, 8 \%, 10 \%)$ and transverse $(0 \%, 1 \%$, $2 \%, 3 \%, 4 \%$ ) slopes, intercepted flow $Q_{\text {int }}$ was measured. Water levels next to the inlet (immediately upstream) were measured too. These experimental data were compared to the data obtained by the 3D calculations. 

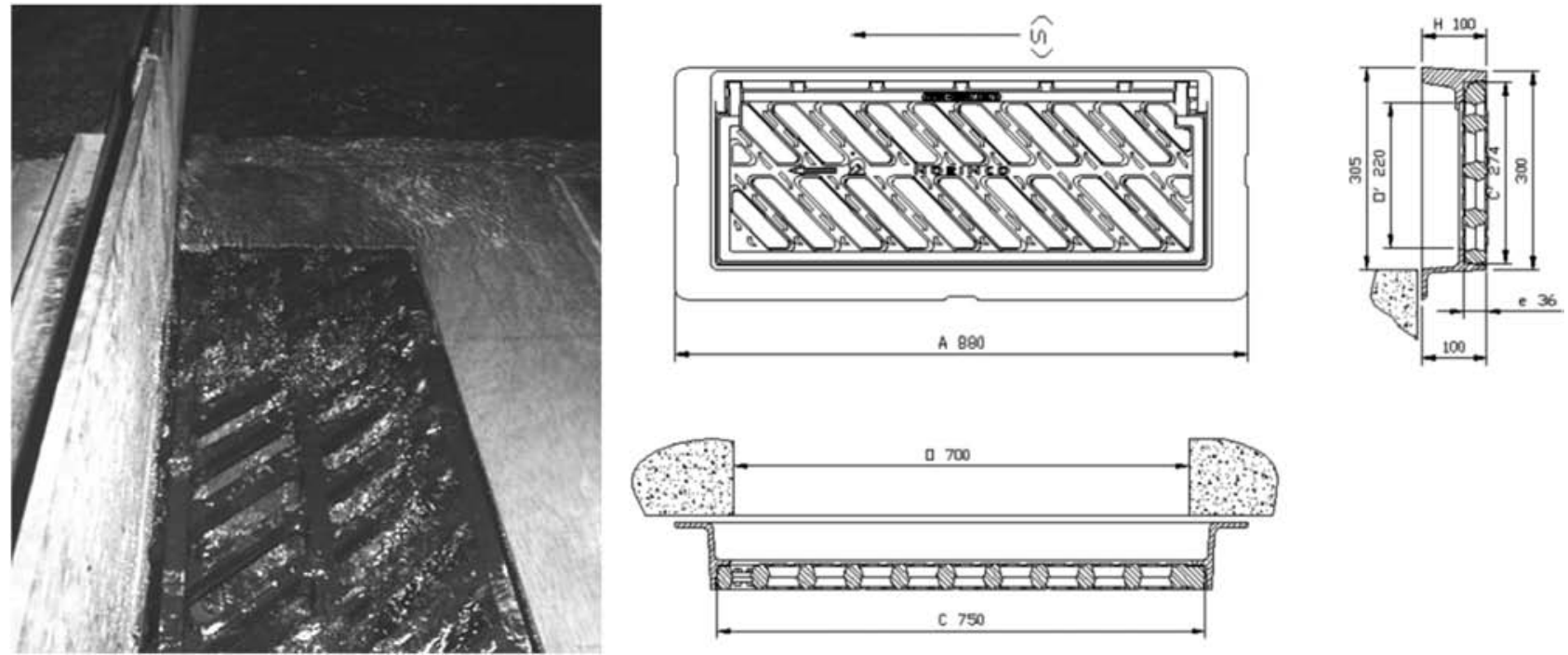

Figure 1 | Photograph and geometric definition of the grate 'Barcelona1'.
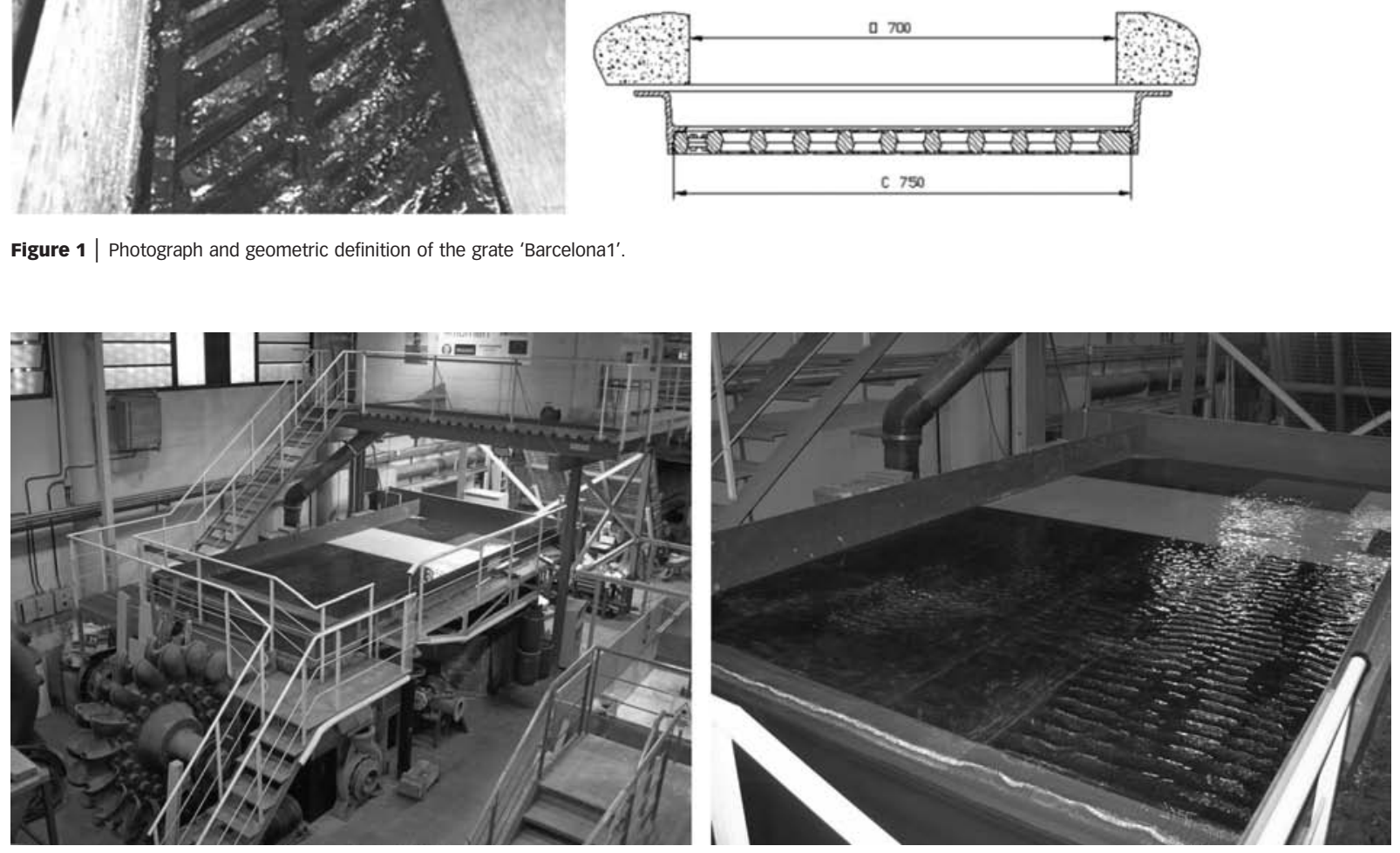

Figure 2 | Photographs of the experimental platform.

\section{NUMERICAL MODEL}

The computer code used for the simulation is Flow-3D. It is a commercial code developed by Flow Science Inc., of the type called CFD (computational fluid dynamics) for a general three-dimensional analysis. The threedimensional numerical modeling developed through Flow-3D code is used for numerous applications by incorporating a 'multiphysics' environment (considering different types of fluids) and especially suited for simulation of free surface flows. The numerically solved equations by Flow-3D for hydraulic studies are mass and momentum conservation equations with some additional terms:

$$
\begin{gathered}
\frac{\partial}{\partial x}\left(u A_{x}\right)+R \frac{\partial}{\partial y}\left(v A_{y}\right)+\frac{\partial}{\partial z}\left(w A_{z}\right)+\xi \frac{u A_{y}}{x}=\frac{R_{S O R}}{\rho} \\
\frac{\partial u}{\partial t}+\frac{1}{V_{F}}\left\{u A_{x} \frac{\partial u}{\partial x}+v A_{y} R \frac{\partial u}{\partial y}+w A_{z} \frac{\partial u}{\partial z}\right\}-\xi \frac{A_{y} v^{2}}{x V_{F}} \\
=-\frac{1}{\rho} \frac{\partial p}{\partial x}+G_{x}+f_{x}-b_{x}-\frac{R_{s o r}}{\rho V_{F}}\left(u-u_{w}-\delta u_{s}\right) \\
\frac{\partial v}{\partial t}+\frac{1}{V_{F}}\left\{u A_{x} \frac{\partial v}{\partial x}+v A_{y} R \frac{\partial v}{\partial y}+w A_{z} \frac{\partial v}{\partial z}\right\}+\xi \frac{A_{y} u v}{x V_{F}} \\
=-\frac{1}{\rho}\left(R \frac{\partial p}{\partial y}\right)+G_{y}+f_{y}-b_{y}-\frac{R_{s o r}}{\rho V_{F}}\left(v-v_{w}-\delta v_{s}\right)
\end{gathered}
$$




$$
\begin{aligned}
& \frac{\partial w}{\partial t}+\frac{1}{V_{F}}\left\{u A_{x} \frac{\partial w}{\partial x}+v A_{y} R \frac{\partial w}{\partial y}+w A_{z} \frac{\partial w}{\partial z}\right\} \\
& =-\frac{1}{\rho} \frac{\partial p}{\partial z}+G_{z}+f_{z}-b_{z}-\frac{R_{\text {sor }}}{\rho V_{F}}\left(w-w_{w}-\delta w_{s}\right)
\end{aligned}
$$

where (2) is the continuity Equations (3)-(5) are the NavierStokes equations, with $u, v$ and $w$ being the velocity components in the $x, y$ and $z$ directions respectively. In addition Flow-3D adds some components to the equations:

- $A_{i}$ : Fractional area open to flow in the i direction

- $R$ : coefficient used to change Cartesian to cylindrical coordinates

- $\xi$ : For Cartesian coordinates this has a value of 0

- $R_{S O R}$ : Mass source. You can add flow entries for example

- $V_{F}$ : Fraction volume of fluid

- $u_{w}, \delta u_{s}$ : Components of relative velocities related to the mass source. For this study are not taken into account

- $G_{i}$ : Part of the gravity acceleration in the i direction

- $f_{i}$ : Component of the viscous forces in the i direction

- $b_{i}$ : A factor that is only taken into account when there are porous media. It was 0 in calculations of this study.

The code solves the 3D Reynolds Averaged Navier Stokes equations (RANS) with a numerical scheme considering finite volumes (Savage \& Johnson 200I). The flow domain is subdivided using Cartesian coordinates considering a 3D mesh composed by variable-sized hexahedral cells, with smaller dimensions in the inlet zone. In a first analysis, this type of network may be considered as a limitation, but it is advantageous because the process to create the mesh is relatively easy and less memory is needed using the tools, VOF and FAVOR ${ }^{\mathrm{TM}}$, in Flow-3D.

For each cell, the numerical scheme considers the values for pressure and velocity at discrete times as indicated in the user's manual. The grid assumes that all variables are calculated at the geometrical centre of each cell (hexahedrons), except the velocities and fractional areas in the boundary cells. The last two are considered in the centre of the cell faces normal to each direction. All terms of the RANS equations are computed from the current time-step values of each variable with an explicit numerical scheme, although other implicit options are available as indicated in the technical documents of Flow-3D. The explicit approach generates an efficient numerical scheme that is more easy to program, but requires a limited time-step value to ensure the stability and accuracy of the results, as happens in all explicit numerical schemes.
If a mesh with square or rectangular sides is used, it is difficult to define curved elements. One possibility is to define a precise mesh with a very small dimension for the hexahedrons and so produce a soft contour, but this incurs a high computational cost.

In order to solve the RANS equations, Flow-3D adopts a finite volume method using a finite difference method plus a FAVOR $^{\mathrm{TM}}$ (Fractional Area and Volume Obstacle Representation) method (Figure 3). These are used to define the different user defined geometrical regions within the grid considered, determining the ratio of area and volume of each cell (Flow-3D 2OII). The concept behind FAVOR ${ }^{\mathrm{TM}}$ is that numerical algorithms are based on information consisting of only one pressure, one velocity, and one temperature, for each control volume, so it would be inconsistent to use more information to define the geometry. Thus, the FAVOR $^{\mathrm{TM}}$ technique retains the simplicity of rectangular elements while representing complex geometric shapes at a level consistent with the use of averaged flow quantities within each volume element (Flow-3D 20II). Computer code does not have yet a compiled version using GPU so in most cases CPU time could be days.

The other algorithm used to define the surface of the calculation mesh is the so-called VOF technique, which was derived from FAVOR ${ }^{\mathrm{TM}}$ and presented by Hirt \& Nichols (I98I). The concept is to follow and capture the interface between two different phases (water and air herein). The VOF method (Figure 4) consists of three ingredients: a

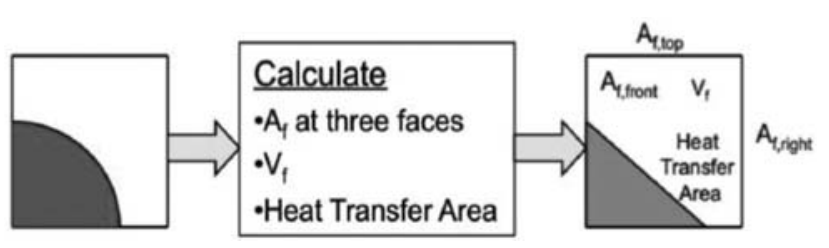

Figure 3 | Scheme of FAVOR ${ }^{\text {TM }}$ method.
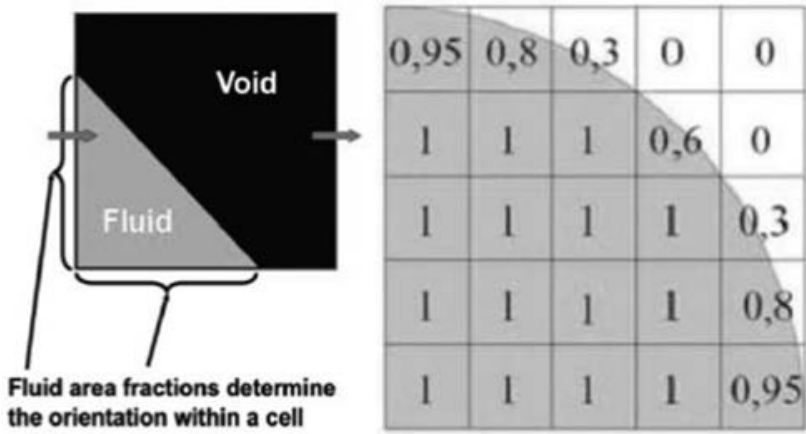

Figure 4 | Scheme of VOF method. 
scheme to locate the surface, an algorithm to track the surface as a sharp interface moving through a computational grid considering a fixed Eulerian reference, and a means of applying boundary conditions at the surface. This algorithm assigns a value between 0 and 1 at each cell depending on the portion of cell is occupied by the water. Cells with the value 1 are completely filled by water while cells with the value 0 are fully occupied by air and finally, cells with intermediate values are interface cells (Ubbink 1997).

One advantage of the VOF method over other methods that define the free surface is that in the VOF method the mesh remains fixed and therefore there is no mesh deformation. This makes the computer algorithms easier, providing better CPU performance and therefore a lower computational cost, although it is still high.

The turbulence model, chosen among all the possibilities included in Flow-3D, is the RNG (Renormalized Group) (Yakhot \& Orszag I986; Yakhot \& Smith 1992). It applies probabilistic methods to the derivatives of the equations describing the amount of turbulence, as turbulent kinetic energy and rate dissipation. This model uses equations similar to the $k-\varepsilon$ model, though constants of the equations in the k- $\varepsilon$ model are empirical while in the RNG model they are explicitly derived.

The choice of this turbulence model was made after a calibration between the physical and numerical model in which several turbulence models, such as RNG, $K-\varepsilon$ and LES (Large Eddy Simulation) were tested in order to achieve the best of both numerical accuracy and computational cost.

According to Figure 5, all the turbulence models give similar results with very small variations (in the order of $1 \%$ among all three). In this sense, even though of these three turbulence models could be used, the RNG model was finally chosen because of both the lowest computational cost and oscillations, once reached the steady flow in the tests made. The calibration process to adapt the 3D model to match the observed data needs to fit the absolute roughness too. The absolute roughness to be included in momentum equations was $K=0.5 \mathrm{~mm}$ for the inlet and for the platform $K=0.9 \mathrm{~mm}$. This set of values produced the best results, minimizing the error between observed and computed values.

The defined mesh is composed of 1,336,440 cells representing $2.57 \mathrm{~m}^{3}$ of simulated volume (real scale). Mesh blocks with an element size of $2 \mathrm{~cm}$ for the main part of the platform and a mesh block with cells of $1 \mathrm{~cm}$ in the vicinity of the inlet were used. Increasing precision near to the inlet allow us to increase the precision of the intercepted flow and therefore of the model results. The $1 \mathrm{~cm}$ cell size has been established according to the geometry of the inlet. With the FAVOR ${ }^{\mathrm{TM}}$ algorithm, it is possible to see how the program interprets the 'Barcelona1' inlet.

In Figure 6, the on the left image represents the inlet geometry sketch while in the views on the right it is possible to preview the geometry after running the FAVORizer Flow3D tool with cell sizes of 2, 1.5, 1 and $0.5 \mathrm{~cm}$. A minimum of $1 \mathrm{~cm}$ in terms of cell size is needed for a good interpretation of this geometry. Several simulations considering these geometries also confirm that this selection was correct. So near the inlet where a best detail of the flow structure is required, cubic cells of $1 \mathrm{~cm}$ were used, while the farther mesh is coarser and cell size increases from 2 up to $4 \mathrm{~cm}$.

Despite Figure 2, in which it is possible to see that initially the platform is empty in the model, the initial condition of the simulation, from now on, has been modified.

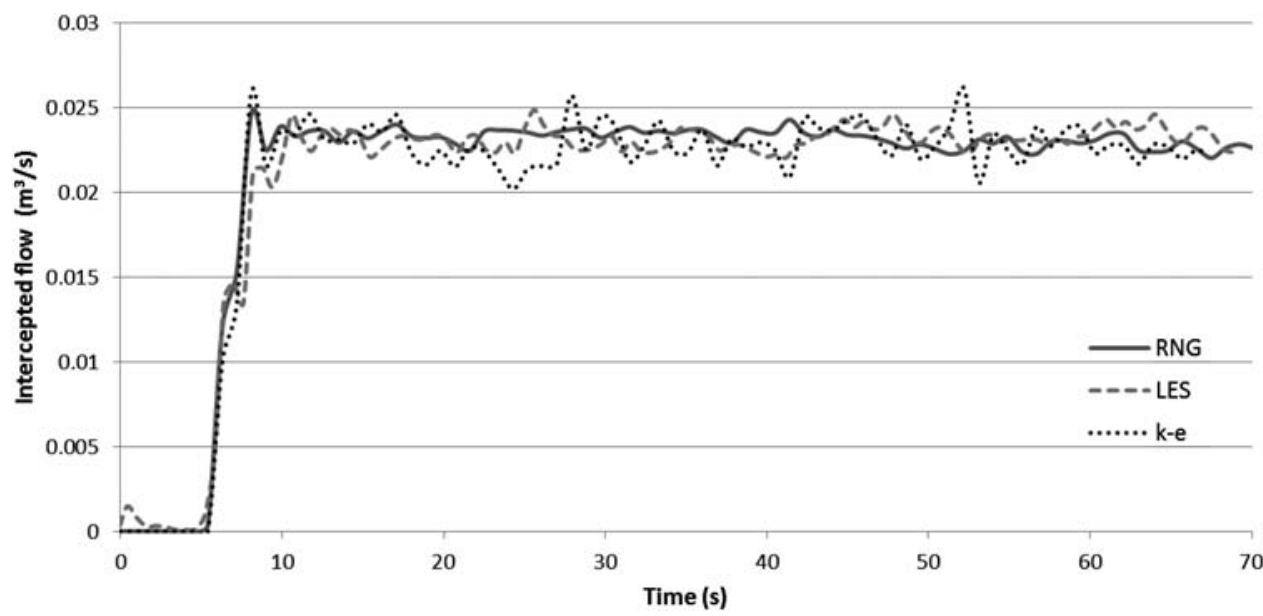

Figure 5 Evolution of the intercepted flow for the same conditions $\left(Q=100 \mathrm{l} / \mathrm{s} \mathrm{S}_{\mathrm{L}}=2 \% \mathrm{~S}_{\mathrm{t}}=2 \%\right)$ and different turbulence models 

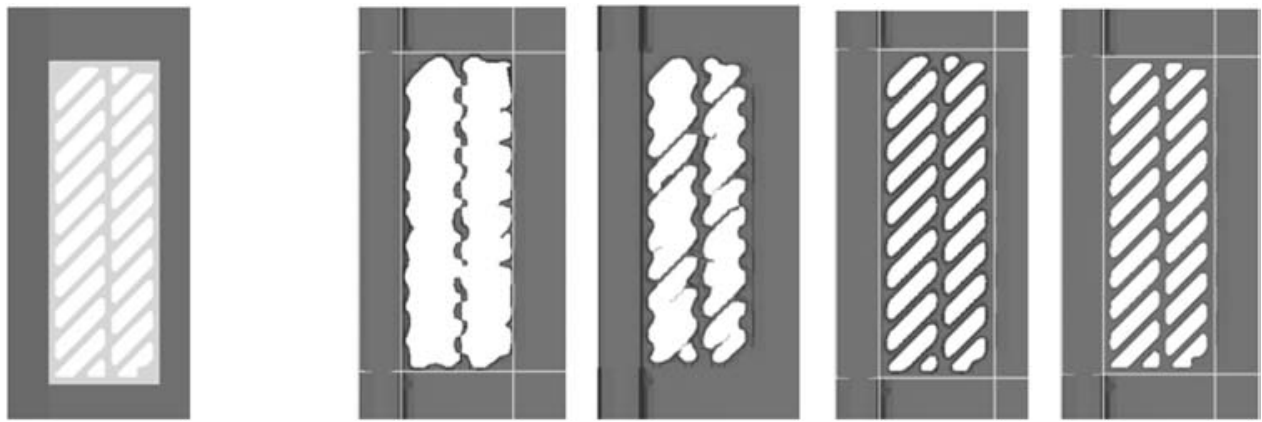

Figure 6 | 'Barcelona1' inlet obtained with FAVOR ${ }^{\mathrm{TM}}$ method considering different mesh sizes $(2,1.5,1$ and $0.5 \mathrm{~cm})$, (real image on the left).

In the experimental set up in laboratory, the model starts with the platform empty, and it fills progressively to reach a steady flow. In the numerical model the simulation starts with the platform partially filled with water. This has been done to decrease the computer time and because the results of this study consider only steady flow conditions.

The modelled geometry in the 3D code is described in Figure 7. The upstream boundary condition is the inflow $Q$ (1/s) arriving at the upstream tank and approaching the grate, while the downstream boundary is a free fall, in most cases in supercritical conditions.

\section{COMPARISON OF EXPERIMENTAL AND NUMERICAL RESULTS}

Different setups (longitudinal and transverse slopes) with the same inlet (Barcelona1) were considered in this study to analyse the interaction between surface flow and pipe flow, and specifically the flow interception phenomenon through the grate. In all cases, the flow captured by the inlet is not influenced by other conditions, so all the flow enters through the inlet with no restrictions that could appear due to the pressure flow in the sewer systems.

Computer time is currently a limiting drawback of the numerical analysis. The average time of simulation oscillates around 2 or 3 days using a computer, Intel Core TM with 2 CPU, $2.3 \mathrm{GHz}$ and $2.99 \mathrm{~Gb}$ RAM. A more powerful workstation or the use of GPU's could reduce the computational time but nowadays there is no commercial version of the software including this feature.

Figure 8 shows a comparison graph between observed and simulated intercepted flow for different inflows (from 25 to $200 \mathrm{l} / \mathrm{s}$ ) and for the same slope of $2 \%$ both in transverse $\left(S_{T}\right)$ and longitudinal $\left(S_{L}\right)$ planes. The solid line represents the results of the numerical model while the

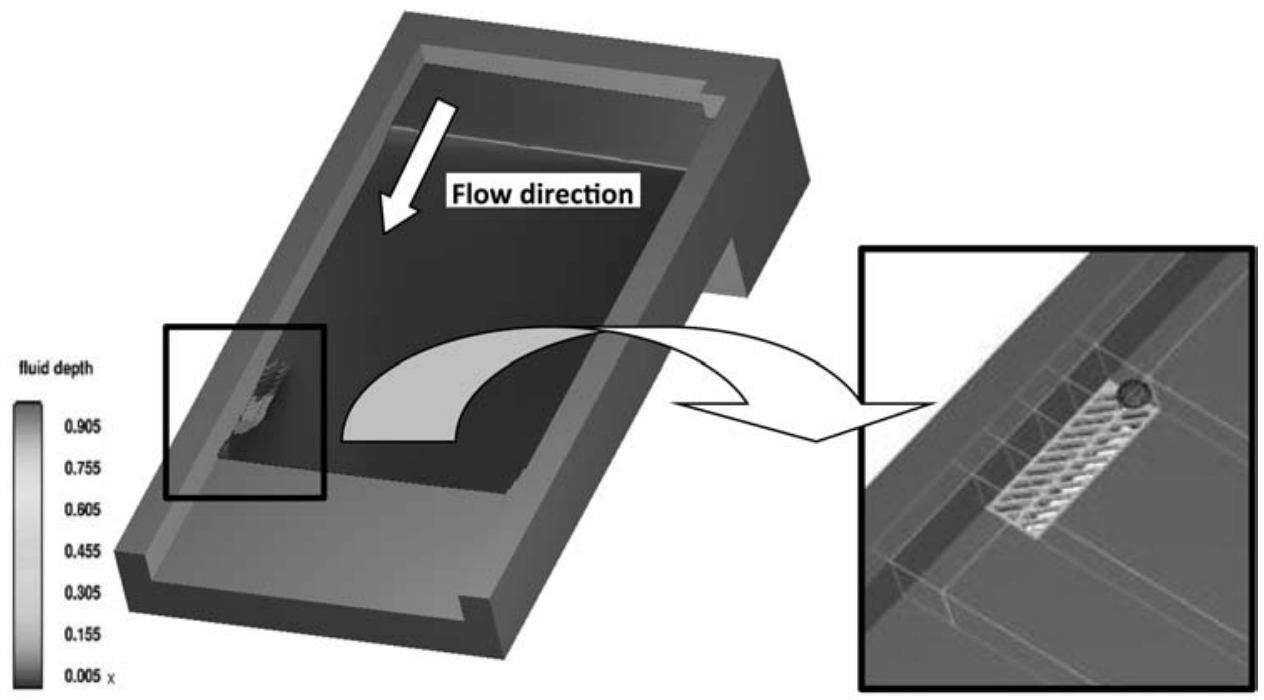

Figure 7 | Platform geometry modelled by Flow-3D and inlet detail. 


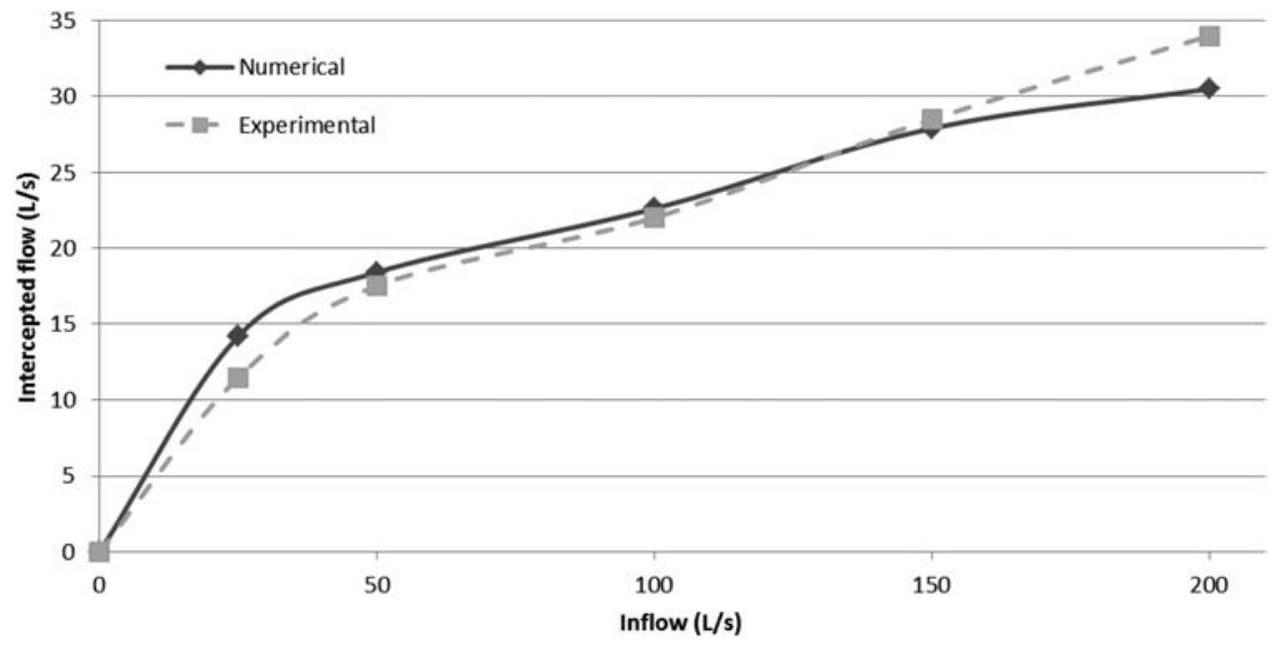

Figure 8 Comparison graph of observed and simulated intercepted flows for different inflows, $S_{L}=2 \%$ and $S_{T}=2 \%$.

broken line shows the experimental measurements. This form of presentation is used in Figures 8 and 9.

Small irregularities in the platform can produce non negligible modifications in flow patterns for low flows (25 l/s). This consideration could explain the relative error (23\%) found in the $25 \mathrm{l} / \mathrm{s}$ simulation although the absolute error is only $3 \mathrm{l} / \mathrm{s}$. On the other hand, for flows of $150 \mathrm{l} / \mathrm{s}$ and above, the error reaches $10 \%$ of measured collected flow and again absolute errors are only $3.5 \mathrm{l} / \mathrm{s}$. This could be due to the characteristics of the upstream tank. It is not large enough to dissipate all the energy introduced by the injection of high flows and turbulence prevents the generation of a complete one-direction flow condition upstream the inlet and distorts the accuracy of results.

In order to assure a good response of the simulation working with high inflows, it will be convenient to simulate a deeper upstream tank and a larger platform. In this way, clearer one-direction flow behaviour upstream of the inlet could be achieved, at the cost of increasing the computational time. However, the object of this study is not to generate the best simulation but to reproduce as accurately as possible the experimental set up previously used.

It can be seen that for low input flows, the simulation model captures more water than observed in the experiments, while for high flow rates the opposite happens. In any case, excluding the extreme flows, the simulated values fit adequately the experimental results (errors of $4 \%$ or less of the total collected flow). Moreover, in terms of absolute errors the differences are usually lower than 1 to $21 /$ s. A similar graph for water depths immediately upstream the inlet can be obtained (Figure 9). In this graph, it is possible to observe an error of a few millimetres

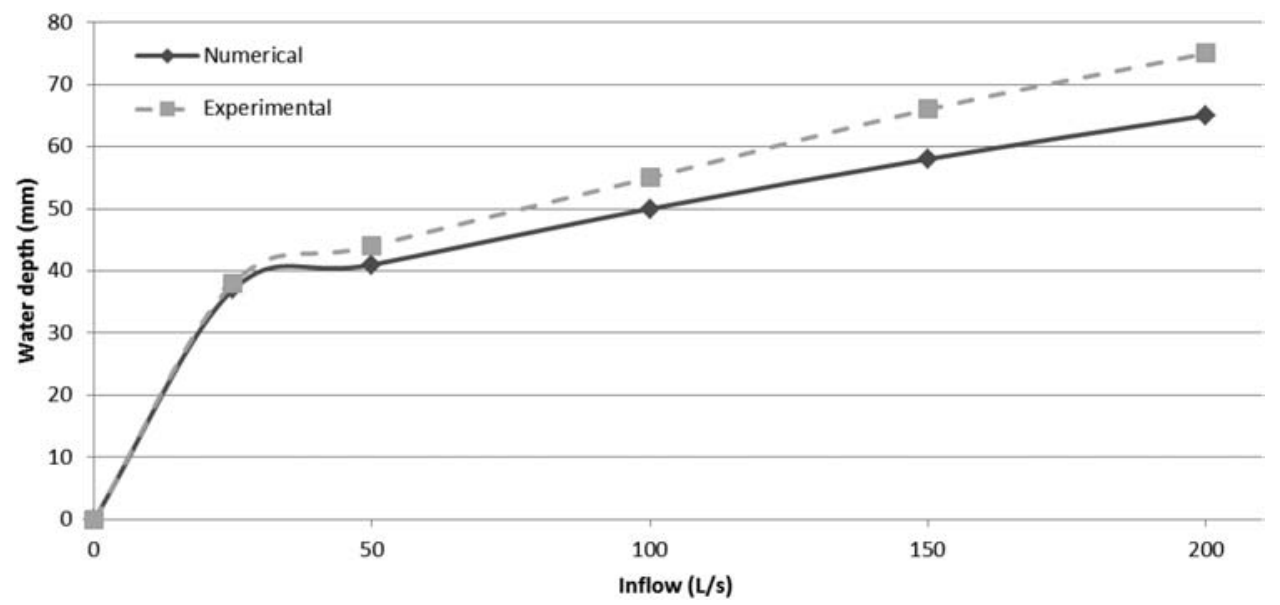

Figure 9 | Comparison graph of water depth upstream the inlet. 
in absolute values, and less than $10 \%$ of measured water depth for relative values.

Taking into account the accuracy of the results of both models, the match between observed and simulated depths upstream of the inlet, the only place where ' $y$ ' was measured, is quite good. As a matter of fact, the measurements in the physical model are made with a millimetre ruler $(1 \mathrm{~mm}$ precision) and the cells of the numerical model, which are $2 \mathrm{~cm}$ height just before the inlet and $1 \mathrm{~cm}$ height within the inlet (the measurement was taken in the border of this two mesh blocks), had a 1-2 mm precision (with VOF code). As a general trend numerical results underestimate the water levels but less than $1 \mathrm{~cm}$ in all cases.
In Figures 10 and 11, data for different combinations of slopes $S_{L}, S_{T}$ and the same inflow of $100 \mathrm{l} / \mathrm{s}$ for both observed and simulated flows are shown. Reference ' $0-2$ ' means $S_{L}=0 \%$ and $S_{T}=2 \%$. Minor errors were observed in intercepted flow, and depth errors are consistent with the accuracy of the depths obtained. The error slightly increases for large longitudinal slopes as can be observed in simulations 6-2 and 10-2.

Furthermore, numerical analysis allows us to obtain more information from each simulation. In this study it was proposed to study the captured water, distinguishing between the frontal and the lateral contribution to the intercepted flows.

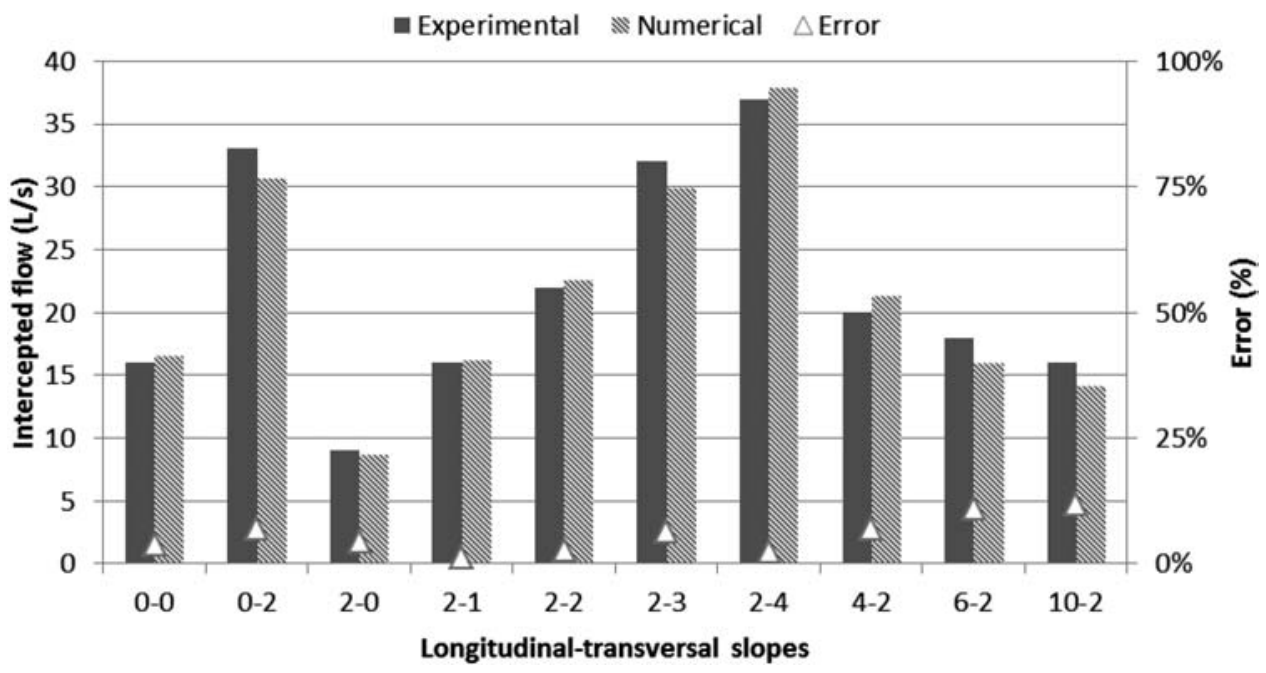

Figure 10 | Diagram of observed and simulated intercepted flows for different combination of $S_{L}$ and $S_{T}$.

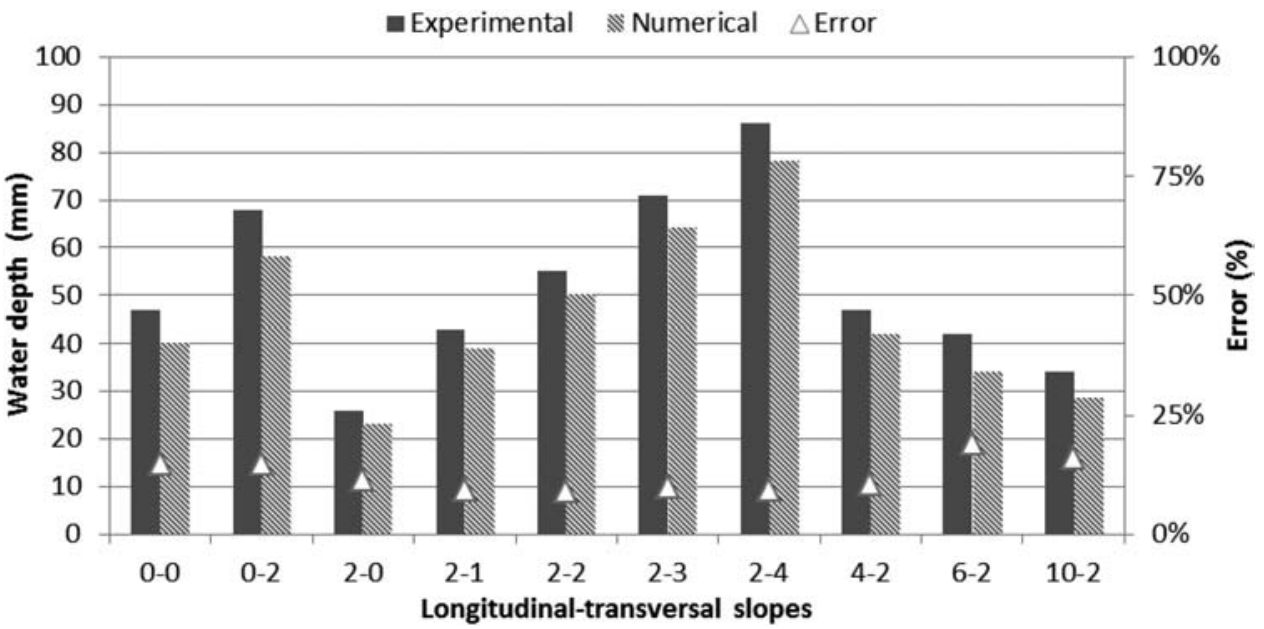

Figure 11 Diagram of observed and simulated flow depth upstream the inlet for different combinations of $S_{L}$ and $S_{T}$. 


\section{Figure 12 | Flow over the inlet for 2\% longitudinal and transversal slopes and USING THE 3D MODEL}

As a first positive conclusion we can state that 3D model could be good enough to approach the inlet efficiency of the inlets, in case they cannot be tested. But from the results obtained in the numerical analysis, 3D simulations provide much more information: flow patterns and structures, and one additional numerical result the estimation of the difference between frontal and lateral flows intercepted by the grate. Looking at the flow structure over the inlet as a result of Flow-3D computation in Figure 12, we can observe that, moving downstream along the inlet, the frontal flow decreases while the lateral increases. It should be remarked that part of the inlet is not in contact with water in the last centimetres of the grate as shown in Figure 12.

Indeed, at the beginning of the inlet the flow is still strongly one-dimensional. As flow moves downstream, the water level decreases and the lateral velocity field increases. For instance, for a simulation of $Q=100 \mathrm{l} / \mathrm{s}, S_{T}=2 \%$ and $S_{L}=2 \%$, along the grate, at the beginning the initial cross velocity is $0.112 \mathrm{~m} / \mathrm{s}$ whereas at the end, cross velocity is $0.456 \mathrm{~m} / \mathrm{s}$, i.e. there is an increase of more than $300 \%$ of the transverse velocity along the lateral boundary of the grate. For the $S_{L}=2 \%$ and $S_{T}=2 \%$ geometry and different flows tested, it is possible to distinguish the percentage of the intercepted flow corresponding to the frontal and lateral part (Figure 13). This distribution varies from $65 \%-35 \%$ for low flow $25 \mathrm{l} / \mathrm{s}$ to $70 \%-30 \%$ for the highest flow of $200 \mathrm{l} / \mathrm{s}$. This information would be much more complicated to obtain in the laboratory because to measure both flows, some instruments or sensors would have to be installed over the grate, which could influence the hydraulics of the collection process.
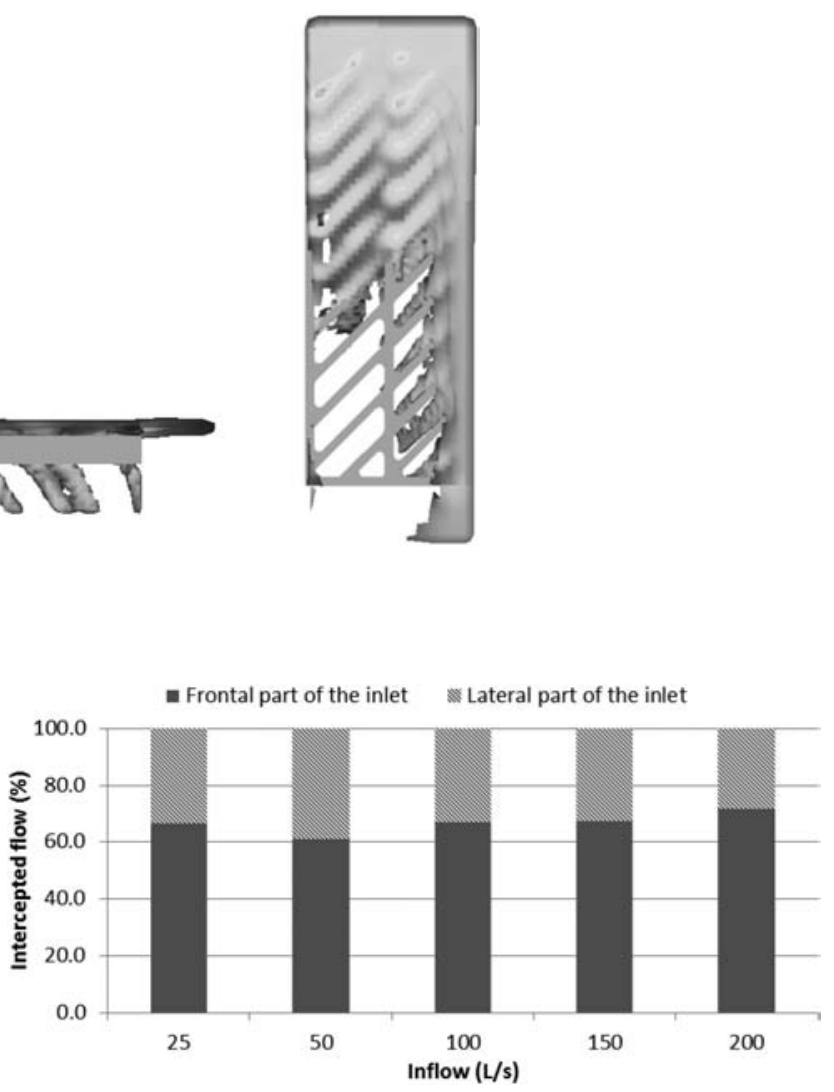

Figure 13 Diagram of the split between frontal (solid) and lateral (crosshatched) intercepted flow for different circulating flows and $S_{L}=2 \%$ and $S_{T}=2 \%$.

Other combinations of longitudinal and transverse slopes have been run in order to compare the frontal and lateral contribution to the intercepted flow. Test cases are indicated in Table 1.

For different setups, the percentage of distribution between frontal and lateral flows oscillates mostly between 60\%-40\% and 80\%-20\% (Figures 13 and 14). The greater the longitudinal slope, the higher the proportion associated with frontal flow. With relatively small slopes (e.g. cases $0-0$ and $0-2$ with $Q=100 \mathrm{l} / \mathrm{s}$ ), a backwater contribution appears in the downstream part of the grate and consequently the water enters into the grate from all three sides. This analysis is not so much relevant for the design of grates but mostly to assess the ability of the turbulence model to replicate

Table 1 | Test cases simulated with 3D flow approach for a circulating flow of $100 \mathrm{l} / \mathrm{s}$

\begin{tabular}{lllllllllll}
\hline Longitudinal slope [\%] & $\mathbf{0}$ & $\mathbf{0}$ & $\mathbf{2}$ & $\mathbf{2}$ & $\mathbf{2}$ & $\mathbf{2}$ & $\mathbf{2}$ & $\mathbf{4}$ & $\mathbf{6}$ & $\mathbf{1 0}$ \\
Transverse slope [\%] & 0 & 2 & 0 & 1 & 2 & 3 & 4 & 2 & 2 & 2 \\
\hline
\end{tabular}




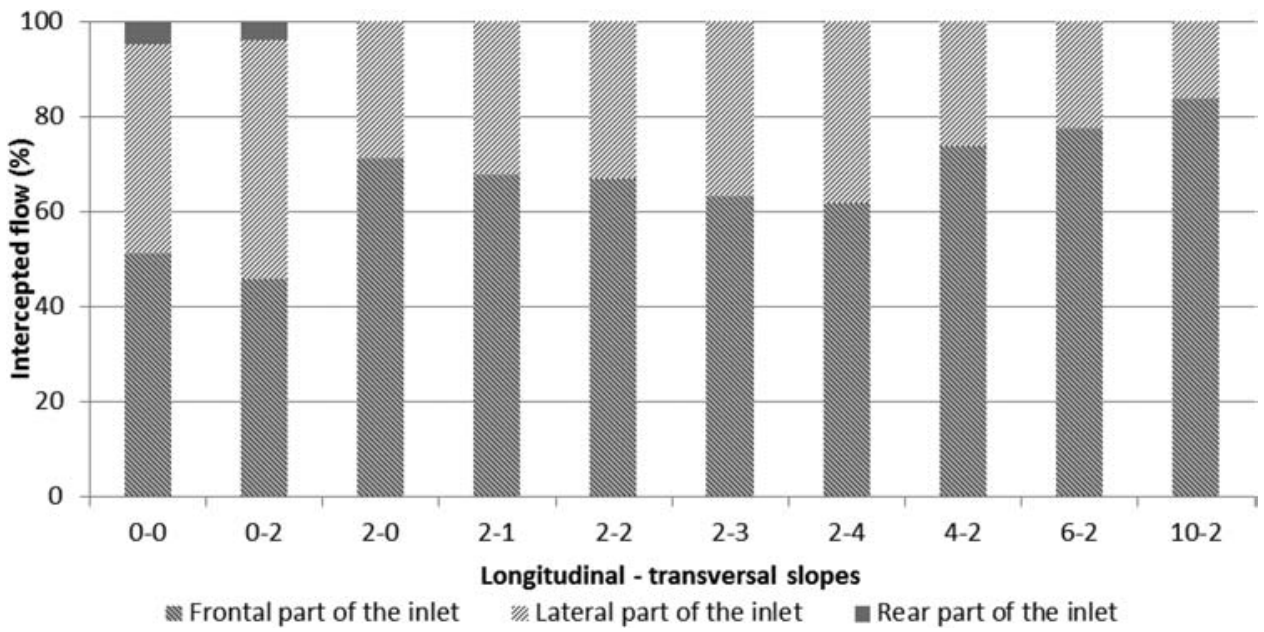

Figure 14 Diagram of the distribution between frontal, lateral and rear intercepted flow, for $Q=100 \mathrm{l} / \mathrm{S}$ and different combination of $S_{L}$ and $S_{T}$.

complex flow features that can be appreciated in the physical model too.

\section{CONCLUSIONS}

From the obtained numerical outputs, we can conclude that three-dimensional modelling is a real alternative to laboratory tests, providing moreover, additional useful information. Differences between numerical and experimental values are acceptable in most cases. So, inlet grates that cannot be tested in a laboratory, can be studied with the 3D model, to approach its efficiency.

Once validated, the numerical model can differentiate and quantify the frontal, side and rear flows approaching the inlet. It is possible to appreciate how this distribution varies with different combinations of inflows and longitudinal and transverse slopes.

It has been observed that, for the grate 'Barcelona1' and any combination of approaching flow and slopes, both $S_{L}$ and $S_{T}$, within the scope of this study, the frontal interception is the main mechanism to capture water, representing $60 \%$ to $80 \%$ of the total water collected. This interception occurs mostly in the first half of the inlet while the lateral collection is distributed almost evenly along the inlet except in the first quarter of the length, a transition zone where the flow changes from a one-dimensional behaviour to a clearer two and three-dimensional pattern.

The results of three-dimensional numerical modelling are then not only a real alternative to laboratory tests to estimate the inlet efficiency, but can even obtain key data of flow patterns that cannot be always easily seen or measured in physical models. This could help in the design of new inlet models. The only drawback at this time is the computational time. Each numerical test takes around 2 or 3 days in the average desktop PC used. Experimental analysis for one grate in the platform located at the laboratory takes 5 days, for the 200 tests usually conducted to characterize the grate according to the undertaken protocol, combinations of 5 different flows, 8 different longitudinal slopes and 5 different transverse slopes. Improvements in CPU and GPU technology could reduce this time drastically, making the use of this $3 \mathrm{D}$ numerical approach more attractive in the near future.

\section{ACKNOWLEDGEMENTS}

This work has been developed in the project CGL201126958/BTE, financed by the Spanish Ministry of Economy and Competitiveness.

\section{$\overline{\text { REFERENCES }}$}

Argue, J. 1986 Storm Drainage Design in Small Urban Catchments. A Handbook for Australian Practice. South Australia Institute of Technology, Adelaide, Australia.

Djordjević, S., Saul, A. J., Tabor, G. R., Blanksby, J., Galambos, I., Sabtu, N. \& Sailor, G. 2013 Experimental and numerical investigation of interactions between above and below ground drainage systems. Water Science \& Technology 67 (3), 535-542.

FHwA 1984 Design of Urban Highway Drainage. Office of Technology Applications, Washington, DC, USA.

FHwA 200I Urban Drainage Design Manual-HEC22. Washington, DC, USA 
FLOW-3D 20II User Manual, Version 10.0. Flow Science, Inc.

Gómez, M. \& Russo, B. 2oII Methodology to estimate hydraulic efficiency of drain inlets. Proceedings of the ICE-Water Management 164 (2), 81-90.

Gómez, M., Hidalgo, G. \& Russo, B. 2013 Experimental campaign to determine grated inlet clogging factors in an urban catchment of Barcelona. Urban Water 10 (1), 51-60.

Hirt, C. W. \& Nichols, B. D. I98I Volume of fluid (VOF) method for the dynamics of free boundaries. Journal of Computational Physics 39, 201-225.

Li, W. H. 1956 The Design of Stormwater Inlets. Johns Hopkins University, Baltimore, MD, USA.

Lopes, P., Leandro, J., Carvalho, R. F., Páscoa, P. \& Martins, R. 2013 Numerical and experimental investigation of a gully under surcharge conditions. Urban Water Journal 13 (6), 468-476.

Savage, B. M. \& Johnson, M. C. 200I Flow over ogee spillway: physical and numerical model case study. J. Hydraulic Eng., ASCE 127 (8), 640-649.
Schmitt, T. G., Thomas, M. \& Ettrich, N. 2004 Analysis and modeling of flooding in urban drainage systems. Journal of Hydrology 299 (3-4), 300-311.

Smagorinsky, J. I963 General circulation experiments with the primitive equations. Mon. Wea. Rev. 91 (3), 99-164.

Spaliviero, F. \& May, R. W. P. 1998 Spacing of road gullies. Hydraulic performance of BS EN124 gully gratings. HR Wallingford, UK.

ten Veldhuis, J. A. E. \& Clemens, F. H. L. R. 201 The efficiency of asset management strategies to reduce urban flood risk. Water Science \& Technology 64 (6), 1317-1324.

Ubbink 1997 Numerical Prediction of Two Fluid Systems with Sharp Interfaces. PhD Thesis. Imperial College of Science, Technology \& Medicine, London.

Yakhot, V. \& Orszag, S. A. I986 Renormalization group analysis of turbulence. I. Basic theory. J. Sci. Comput. 1 (1), 3-51.

Yakhot, V. \& Smith, L. M. 1992 The renormalization group, the eexpansion and derivation of turbulence models. J. Sci. Comput. 7 (1), 35-61.

First received 4 November 2015; accepted in revised form 15 June 2016. Available online 9 August 2016 


\section{Author Queries}

\section{Journal: Water Science \& Technology \\ Manuscript: WST-EM151595R3}

Q1 Please provide name of the city for affiliations.

Q2 Please provide keywords.

Q3 Veldhuis \& Clemens (2011) has been changed to ten Veldhuis \& Clemens (2011) as per the reference list.

Q4 Spaliviero et al. (1998) has been changed to Spaliviero and May (1998) as per the reference list.

Q5 Gómez et al. (2011) has been changed to Gómez and Russo (2011) as per the reference list.

Q6 Djordjevic et al. (2013) has been changed to Djordjević et al. (2013) as per the reference list.

Q7 FHwA (1984) is not cited in the main text. Please confirm where it should be cited, or delete the reference.

Q8 Please provide name of publication for FhwA (2001).

Q9 FHwA (2001) is not cited in the main text. Please confirm where it should be cited, or delete the reference.

Q10 Smagorinsky (1963) is not cited in the main text. Please confirm where it should be cited, or delete the reference.

Q11 In supplied Figure 1 is not sufficient print quality. Please resupply as a high resolution file (300 dpi or above) with sharp lines and text.

Q12 In supplied Figure 3 is not sufficient print quality. Please resupply as a high resolution file (300 dpi or above) with sharp lines and text.

Q13 In supplied Figure 4 is not sufficient print quality. Please resupply as a high resolution file (300 dpi or above) with sharp lines and text.

Q14 In supplied Figure 5 is not sufficient print quality. Please resupply as a high resolution file (300 dpi or above) with sharp lines and text.

Q15 In supplied Figure 6 is not sufficient print quality. Please resupply as a high resolution file (300 dpi or above) with sharp lines and text.

Q16 In supplied Figure 7 is not sufficient print quality. Please resupply as a high resolution file (300 dpi or above) with sharp lines and text.

Q17 In supplied Figure 8 is not sufficient print quality. Please resupply as a high resolution file (300 dpi or above) with sharp lines and text.

Q18 In supplied Figure 9 is not sufficient print quality. Please resupply as a high resolution file (300 dpi or above) with sharp lines and text.

Q19 In supplied Figure 12 is not sufficient print quality. Please resupply as a high resolution file (300 dpi or above) with sharp lines and text.

Q20 In supplied Figure 14 is not sufficient print quality. Please resupply as a high resolution file (300 dpi or above) with sharp lines and text. 